\title{
SOME INEQUALITIES FOR FOURIER TRANSFORMS
}

\section{ARMEN H. ZEMANIAN}

Let $\phi(t)$ be a real and integrable function on $(0, \infty)$ and consider the Fourier cosine and sine transforms given by (1) through (3),

$$
\begin{aligned}
f(x) & =\int_{0}^{\infty} \phi(t) \cos x t d t, \\
g(x) & =\int_{0}^{\infty} \phi(t) \sin x t d t \\
F(x) & =\int_{0}^{x} f(\xi) d \xi=\int_{0}^{\infty} \frac{\phi(t)}{t} \sin x t d t .
\end{aligned}
$$

Then $f(x)$ is even, and $g(x)$ and $F(x)$ are odd. So only the interval $0 \leqq x$ is considered. From (1)

$$
f(0)=\int_{0}^{\infty} \phi(t) d t .
$$

Moreover, if it is assumed that $\phi(t)$ is of bounded variation in the interval $(0, \epsilon)$, then from (3)

$$
F(\infty)=\phi(0+) \frac{\pi}{2} .
$$

This expression follows readily from a known theorem on Fourier transforms [1, Theorem 3, p. 13] if $\phi(t)$ is defined in the interval $(-\infty, 0)$ by the relation $\phi(-t)=\phi(t)$.

In this paper an inequality on $f(x)$ for monotonic decreasing $\phi(t)$ and inequalities on $g(x)$ and $F(x)$ for non-negative $\phi(t)$ are obtained when the bounds on these functions are known for $x \geqq 1$. The result on $f(x)$ is similar to an inequality obtained by Boas and Kac [2]. In the more general case when $\phi(t) \geqq 0$, they use the more restrictive condition, $f(x)=0$ for $x \geqq 1$, to show that then

$$
|f(x)| \leqq f(0) \cos \frac{\pi}{[1 / x]+1}, \quad 0 \leqq x<1
$$

where $[t]$ denotes the least integer not less than $t$.

The main results depend upon two inequalities that the sine func-

Received by the editors May 4, 1956 and, in revised form, June 27, 1956 and September 19, 1956. 
tion satisfies, which are stated by Lemma 3. Lemmas 1 and 2 will be needed in the proof of the third one.

LEMma 1. If $v(u)$ is an even, periodic and integrable function whose period is $2 \pi y$ where $0 \leqq y<1$, and if $v(u) \geqq \cos u$ for $-\pi y \leqq u \leqq \pi y$, then

$$
\int_{0}^{x} v(u) d u \geqq \sin x \quad \text { for } x \geqq 0 .
$$

Proof. First assume the point $x$ is such that $\cos x \leqq \cos \pi y$. For $n$ equal to zero or a positive integer

$$
\begin{aligned}
\sin x= & \int_{0}^{x} \cos u d u \\
= & \int_{0}^{\pi y} \cos u d u+\int_{\pi y}^{\pi(2-y)} \cos u d u+\int_{x(2-y)}^{\pi(2+y)} \cos u d u \\
& +\int_{x(2+y)}^{\pi(4-y)} \cos u d u+\cdots+\int_{x(2 n+y)}^{x} \cos u d u \\
= & \int_{0}^{(2 n+1) \pi y} g(u) d u+\int_{0}^{x-(2 n+1) \pi y} h(u) d u
\end{aligned}
$$

where $g(u)$ is periodic with a period of $2 \pi y$ and equals $\cos u$ for $-\pi y \leqq u \leqq \pi y$ and $h(u)$ is periodic with a period of $2 \pi(1-y)$ and equals $\cos (u+\pi y)$ for $0 \leqq u \leqq 2 \pi(1-y)$. But $g(u) \leqq v(u)$ and $h(u)$ $\leqq \cos \pi y \leqq v(u)$ for all $u$ by hypothesis. Therefore,

$$
\int_{0}^{(2 n+1) \pi y} g(u) d u \leqq \int_{0}^{(2 n+1) x y} v(u) d u
$$

and,

$$
\begin{aligned}
\int_{0}^{x-(2 n+1) \pi y} h(u) d u & \leqq \int_{0}^{x-(2 n+1) \pi y} \cos \pi y d u=\int_{(2 n+1) \pi y}^{x} \cos \pi y d u \\
& \leqq \int_{(2 n+1) \pi y}^{x} v(u) d u .
\end{aligned}
$$

Thus,

$$
\int_{0}^{x} \cos u d u \leqq \int_{0}^{x} v(u) d u \quad \text { for all } x \geqq 0
$$

The proof proceeds in the same way for the case where $\cos x \geqq \cos \pi y$. 
LEMMA 2. If $w(u)$ is an even, periodic and integrable function whose period is $2 \pi y$ where $0 \leqq y<1$ and if $w(u) \leqq \cos [u+\pi(1-y)]$ for $-\pi y \leqq u \leqq \pi y$, then

$$
\int_{0}^{x} w(u) d u \leqq \sin x \quad \text { for } x \geqq 0 .
$$

Proof. This lemma will be proved for the case where $\cos x$ $\geqq \cos \pi(1-y)$ since the proof proceeds in the same way when $\cos x \leqq \cos \pi(1-y)$. For $n$ a positive integer

$$
\begin{aligned}
\sin x= & \int_{0}^{x} \cos u d u \\
= & \int_{0}^{\pi(1-y)} \cos u d u+\int_{\pi(1-y)}^{\pi(1+y)} \cos u d u+\int_{x(1+y)}^{\pi(3-y)} \cos u d u \\
& +\int_{\pi(3-y)}^{\pi(3+y)} \cos u d u+\cdots+\int_{\pi(2 n-1+y)}^{x} \cos u d u \\
= & \int_{0}^{2 n \pi y} p(u) d u+\int_{0}^{x-2 n \pi y} q(u) d u
\end{aligned}
$$

where $p(u)$ is periodic with a period of $2 \pi y$ and equals $\cos [u+\pi(1-y)]$ for $0 \leqq u \leqq 2 \pi y$ and $q(u)$ is periodic with a period of $2 \pi(1-y)$ and equals $\cos u$ for $-\pi(1-y) \leqq u \leqq \pi(1-y)$. But since $w(u) \leqq p(u)$ and $w(u) \leqq \cos \pi(1-y) \leqq q(u)$,

$$
\int_{0}^{2 n \pi y} p(u) d u \geqq \int_{0}^{2 n \pi y} w(u) d u
$$

and,

$$
\begin{aligned}
\int_{0}^{x-2 n \pi y} q(u) d u & \geqq \int_{0}^{x-2 n \pi y} \cos \pi(1-y) d u=\int_{2 n \pi y}^{x} \cos \pi(1-y) d u \\
& \geqq \int_{2 n \pi y}^{x} w(u) d u .
\end{aligned}
$$

Thus this lemma holds when $x \geqq \pi(1+y)$. For $0 \leqq x \leqq \pi(1+y)$, the proof is trivial.

Lemma 3. For $0 \leqq y<1, x \geqq 0$ and $N$ a positive integer,

$$
\sin x \leqq Q_{0} x+y Q_{2} \sin \frac{x}{y}+\cdots+\frac{y Q_{2 N}}{N} \sin \frac{N x}{y}
$$


(7) $\quad \sin x \geqq-Q_{0} x+y Q_{2} \sin \frac{x}{y}-\cdots+(-1)^{N+1} \frac{y Q_{2 N}}{N} \sin \frac{N x}{y}$

where

$$
\begin{aligned}
Q_{0} & =1+\sum_{k=1}^{N} \frac{\left(-y^{2}\right)\left(1^{2}-y^{2}\right) \cdots\left[(k-1)^{2}-y^{2}\right]}{(k !)^{2}}, \\
Q_{2 p} & =(-1)^{p} 2 \sum_{k=p}^{N} \frac{\left(-y^{2}\right)\left(1^{2}-y^{2}\right) \cdots\left[(k-1)^{2}-y^{2}\right]}{(k-p) !(k+p) !} \\
p=1,2, \cdots, N . &
\end{aligned}
$$

Proof. Consider the expansion of the cosine function given by (10) where $-\pi / 2 \leqq \theta \leqq \pi / 2$ and $m$ is a real number [4, p. 276, Equation 5 ].

$$
\begin{aligned}
\cos m \theta=1 & -\frac{m^{2}}{2 !} \sin ^{2} \theta-\frac{m^{2}\left(2^{2}-m^{2}\right)}{4 !} \sin ^{4} \theta-\cdots \\
& -\frac{m^{2}\left(2^{2}-m^{2}\right) \cdots\left[(2 k-2)^{2}-m^{2}\right]}{(2 k) !} \sin ^{2 k} \theta-\cdots
\end{aligned}
$$

If $m$ is restricted to the interval $0 \leqq m \leqq 2$, then every term after the first is a nonpositive quantity and so $\cos m \theta$ is less than or equal to any finite sum obtained by terminating the right hand side of (10) at any term after the first. Terminating after the $(N+1)$ th term, replacing the powers of the sine function by their expansions in terms of the sums of cosines of multiples of $\theta[4,54$, equation 44$]$ and using the change of variable $y=m / 2$, (11) may be obtained where $0 \leqq y<1$ and the $Q$ 's are given by (8) and (9).

$$
\cos 2 y \theta \leqq Q_{0}+Q_{2} \cos 2 \theta+\cdots+Q_{2 N} \cos 2 N \theta .
$$

Writing $u=2 y \theta$ and then invoking Lemma 1 , (6) will be the result.

Similarly the negative of the function obtained by replacing $\theta$ in the right hand side of (11) by $(\mu / 2 y-\pi / 2)$ satisfies the conditions of Lemma 2 and thereby (7) follows immediately.

The result on $f(x)$ is given by Theorem 1 .

THEOREM 1. If $\phi(t)$ is real, integrable, monotonic decreasing and absolutely continuous in $(0, \infty)$, if $f(x)$ is defined by (1) and if $|f(x)|$ $\leqq k f(0)$ with $0 \leqq k<1$ when $x \geqq 1$, then for $0 \leqq x<1$ :

$$
\frac{|f(x)|}{f(0)} \leqq \frac{\sin \pi x}{\pi x}+k\left[\frac{\sin \pi x}{\pi x}-\cos \pi x\right] .
$$

Proof. Integrating (1) by parts, 


$$
f(x)=-\frac{1}{x} \int_{0}^{\infty} \frac{d \phi}{d t} \sin x t d t .
$$

The limit terms in this integration by parts vanish since $\phi(t)$ is real, integrable and monotonic decreasing in $(0, \infty)$. For $0<\int_{R}^{S} \phi(t) d t<\epsilon$ if $R$ and $S$ are large enough. So $\epsilon>\phi(S) \int_{R}^{S} d t=(S-R) \phi(s)$. Fixing $R$ and letting $S \rightarrow \infty, \lim _{S \rightarrow \infty} S \phi(S)$ is seen to vanish. Similarly $0<\int_{R}^{S} \phi(t) d t$ $<\epsilon$ if $R$ and $S$ are close enough to zero. So $\epsilon>(S-R) \phi(S)$. If $R=S^{2}$, then $\lim _{S \rightarrow 0} S \phi(S)=0$. Thus $\lim \phi(t) x^{-1} \sin x t=0$ for both $t \rightarrow 0$ and $t \rightarrow \infty$.

Replacing $\sin x t$ in the integrand of (13) by the right hand side of (6), bearing in mind that $-d \phi / d t \geqq 0$ almost everywhere, and integrating,

$$
f(x) \leqq Q_{0} f(0)+Q_{2} f\left(\frac{x}{y}\right)+\cdots+Q_{2 N} f\left(\frac{N x}{y}\right) .
$$

Now this inequality holds for all $N$ and so for $N \rightarrow \infty$. Furthermore the $Q$ 's may be summed as follows, when $N \rightarrow \infty$ [3, equation 2.8 (46) and equation $1.2(8)$ ].

$$
\begin{aligned}
Q_{0} \rightarrow q_{0}= & F(y,-y ; 1 ; 1)=\frac{\sin \pi y}{\pi y}, \\
Q_{2 p} \rightarrow q_{2 p}= & (-1)^{p} 2 \frac{\left(-y^{2}\right)\left(1^{2}-y^{2}\right) \cdots\left[(p-1)^{2}-y^{2}\right]}{(2 p) !} \\
& \cdot F(p+y, p-y ; 2 p+1 ; 1) \\
= & (-1)^{p+1} \frac{2 y \sin \pi y}{\pi\left(p^{2}-y^{2}\right)} \quad p=1,2,3, \cdots .
\end{aligned}
$$

Moreover $\phi(t)$ is a non-negative function by hypothesis and so $|f(x)| \leqq f(0)$. This means that the double infinite series represented by $\lim _{N \rightarrow \infty} \sum_{p=0}^{N} Q_{2 p} f(p x / y)$ converges absolutely for $0 \leqq y<1$ as can be seen from the following.

$$
\begin{aligned}
\lim _{N \rightarrow \infty} & \sum_{p=0}^{N} Q_{2 p} f\left(\frac{p x}{y}\right) \\
= & \left\{1+\sum_{k=1}^{\infty} \frac{\left(-y^{2}\right)\left(1^{2}-y^{2}\right) \cdots\left[(k-1)^{2}-y^{2}\right]}{(k !)^{2}}\right\} f(0) \\
& \quad+\sum_{p=1}^{\infty}(-1)^{p} 2 \sum_{k=p}^{\infty} \frac{\left(-y^{2}\right)\left(1^{2}-y^{2}\right) \cdots\left[(k-1)^{2}-y^{2}\right]}{(k-p) !(k+p) !} f\left(\frac{p x}{y}\right) .
\end{aligned}
$$

Replacing each term by its absolute value and $f(p x / y)$ by $f(0)$, a 
double series of positive terms is obtained which converges to the sum of the absolute values of the $q_{2 p}$ multiplied by $f(0)$ as was shown above.

$$
\begin{aligned}
\lim _{N \rightarrow \infty} \sum_{p=0}^{N} Q_{2 p} f\left(\frac{p x}{y}\right) & \leqq f(0)\left[\frac{\sin \pi y}{\pi y}+\sum_{p=1}^{\infty} \frac{2 y \sin \pi y}{\pi\left(p^{2}-y^{2}\right)}\right] \\
& =f(0)\left[2 \frac{\sin \pi y}{\pi y}-\cos \pi y\right] .
\end{aligned}
$$

Thus,

$$
f(x) \leqq \lim _{N \rightarrow \infty} \sum_{p=0}^{N} Q_{2_{p}} f\left(\frac{p x}{y}\right)=\sum_{p=0}^{\infty} q_{2 p} f\left(\frac{p x}{y}\right) .
$$

Since $|f(x)| \leqq k f(0)$ for $x \geqq 1$, and since the $q_{2 p}$ are positive for $p$ odd and negative for $p$ even, the upper bound of (12) may be obtained upon replacing the $f(p x / y)$ by $k f(0)$ for $p$ odd and by $-k f(0)$ for $p$ even when $x / y$ equals 1 and then substituting the infinite series,

$$
\sum_{n=1}^{\infty} \frac{1}{n^{2}-y^{2}}=\frac{1}{2 y^{2}}-\frac{\pi}{2 y} \cot \pi y, \quad 0 \leqq y<1 .
$$

Similarly, replacing $\sin x t$ in (13) by the right hand side of (7), the lower bound of (12) will follow. The double series obtained in this case can again be shown to converge absolutely.

It should be noted that, under the conditions of this theorem, it is obvious that $|f(x)| \leqq f(0)$. For $0 \leqq x<1,(12)$ is an improvement over this result only for

$$
k \leqq \frac{1-(\sin \pi x) / \pi x}{(\sin \pi x) / \pi x-\cos \pi x} .
$$

Similar inequalities may be obtained on $g(x)$ and $F(x)$.

THEOREM 2. If $\phi(t)$ is real, integrable and non-negative on $(0, \infty)$, if $\int_{0}^{\infty} t \phi(t) d t=M$, if $g(x)$ is defined by (2), and if $|g(x)| \leqq k_{1}$ for $x \geqq 1$ where $M$ and $k_{1}$ are positive real numbers, then for $0<x<1$

$$
|g(x)| \leqq \frac{\sin \pi x}{\pi}\left\{M-k_{1}[\Psi(x)+\Psi(-x)+2 \gamma]\right\}
$$

where

$$
\Psi(x)=\frac{d}{d x} \log \Gamma(x)
$$

and 


$$
\gamma=\lim _{m \rightarrow \infty}\left(\sum_{n=1}^{m} \frac{1}{n}-\log m\right)=.577215 \cdots
$$

Proof. Again inserting the right hand side of (6) in place of $\sin x t$ in (2), integrating and then letting $N$ go to infinity, expression (16) may be obtained where $0 \leqq y<1$. Since $\phi(t) \geqq 0,|g(x)| \leqq f(0)$ and so the double series occurring in this case can also be shown to converge absolutely by using the same procedure that was employed in the proof of Theorem 1.

$$
g(x) \leqq q_{0} M x+y q_{2} g\left(\frac{x}{y}\right)+\frac{y q_{4}}{2} g\left(\frac{2 x}{y}\right)+\cdots .
$$

Upon letting $x / y$ equal one, the $g(p x / y)$ in (16) may be replaced by $k_{1}$ for $p$ odd and by $-k_{1}$ for $p$ even. This will yield for $0 \leqq x<1$

$$
g(x) \leqq \frac{\sin \pi x}{\pi}\left[M+2 x^{2} k_{1} \sum_{n=1}^{\infty} \frac{1}{n\left(n^{2}-x^{2}\right)}\right] .
$$

The infinite series in the right hand side of this expression may be expressed in closed form by using a known series for $\Psi(x)[3$, equation $1.7(3)]$.

$$
2 x^{2} \sum_{n=1}^{\infty} \frac{1}{n\left(n^{2}-x^{2}\right)}=-[\Psi(x)+\Psi(-x)+2 \gamma] .
$$

This yields the upper bounds of (15).

The lower bounds are derived in a similar way using (7). In this case each coefficient of the $g(p x / y)$ in the expression corresponding to (16) is positive. So each of the $g(p x / y)$ are replaced by $-k_{1}$ to maintain the inequality.

TheOREM 3. If $\phi(t)$ is real, integrable, and non-negative on $(0, \infty)$ and of bounded variation on $(0, \epsilon)$, if $F(x), f(0)$ and $F(\infty)$ are defined by (3), (4) and (5) respectively, and if

$$
\left(1-k_{2}\right) F(\infty) \leqq F(x) \leqq\left(1+k_{2}\right) F(\infty) \text { for } x \geqq 1
$$

where $k_{2}$ is a positive real number, then for $0<x<1$

$$
\begin{aligned}
F(x) \leqq \frac{\sin \pi x}{\pi}\{f(0)+2 F(\infty) & x^{2} \sum_{n=1}^{\infty} \frac{(-1)^{n+1}}{n\left(n^{2}-x^{2}\right)} \\
& \left.-k_{2} F(\infty)[\Psi(x)+\Psi(-x)+2 \gamma]\right\}
\end{aligned}
$$


(18) $\quad F(x) \geqq-\frac{\sin \pi x}{\pi}\left\{f(0)+\left(1-k_{2}\right) F(\infty)[\Psi(x)+\Psi(-x)+2 \gamma]\right\}$ where $\Psi(x)$ and $\gamma$ are defined as in Theorem 2 .

Proof. This proof is very similar to the proof of Theorem 2. Again (6) is substituted into (3) to obtain the inequality (19) after $N$ is taken to infinity. Once again $|F(x)| \leqq f(0) x$ since $\phi(t)$ is non-negative and therefore the double series converges absolutely as was shown in the proof of Theorem 1 . In this expression, $0 \leqq y<1$.

$$
F(x) \leqq q_{0} f(0) x+y q_{2} F\left(\frac{x}{y}\right)+\frac{y q_{4}}{2} F\left(\frac{2 x}{y}\right)+\cdots .
$$

Then setting $x / y$ equal to one, replacing the $F(p x / y)$ by $\left(1+k_{2}\right) F(\infty)$ for $p$ odd and by $\left(1-k_{2}\right) F(\infty)$ for $p$ even, and using the same infinite series expression for $\Psi(x)$ as before, (17) may be obtained.

The lower bound (18) is obtained by using (7) in a similar fashion. Again each coefficient of the $F(p x / y)$ in the expression corresponding to (19) is positive and so the $F(p x / y)$ are replaced by $\left(1-k_{2}\right) F(\infty)$.

\section{BIBLIOGRAPHY}

1. E. C. Titchmarsh, Introduction to the theory of Fourier integrals, Oxford University Press, 2d ed., 1948.

2. R. P. Boas, Jr. and M. Kac, Inequalities for Fourier transforms of positive functions, Duke Math. J. vol. 12 (1945) pp. 189-206.

3. A. Erdelyi, W. Magnus, F. Oberhettinger, and F. G. Tricomi, Higher transcendental functions, vol. I, McGraw-Hill, 1953.

4. E. W. Hobson, Plane trigonometry, Cambridge University Press, 7th ed., 1939.

NeW YoRK UNIVERSITY 